\title{
Could quantum gravity be tested with high intensity Lasers?
}

\author{
João Magueijo ${ }^{1,2,3}$ \\ ${ }^{1}$ Perimeter Institute for Theoretical Physics, 31 Caroline St N, Waterloo N2L 2Y5, Canada \\ ${ }^{2}$ Canadian Institute for Theoretical Astrophysics, 60 St George St, Toronto M5S 3H8, Canada \\ 3 Theoretical Physics Group, Imperial College, Prince Consort Road, London SW7 2BZ, England
}

(Dated: August 31, 2018)

\begin{abstract}
In quantum gravity theories Planckian behavior is triggered by the energy of elementary particles approaching the Planck energy, $E_{P}$, but it's also possible that anomalous behavior strikes systems of particles with total energy near $E_{P}$. This is usually perceived to be pathological and has been labelled "the soccer ball problem". We point out that there is no obvious contradiction with experiment if coherent collections of particles with bulk energy of order $E_{P}$ do indeed display Planckian behavior, a possibility that would open a new experimental window. Unfortunately field theory realizations of deformed special relativity never exhibit a "soccer ball problem"; we present several formulations where this is undeniably true. Upon closer scrutiny we discover that the only chance for Planckian behavior to be triggered by large coherent energies involves the details of second quantization. We find a formulation where the quanta have their energy-momentum (mass-shell) relations deformed as a function of the bulk energy of the coherent packet to which they belong, rather than the frequency. Given ongoing developments in Laser technology, such a possibility would be of great experimental interest.
\end{abstract}

\section{INTRODUCTION}

Non-linear or deformed special relativity (hereafter DSR) is an interesting arena for studying quantum gravity (QG) phenomenology at energies close to the Planck energy $E_{P}=1 / l_{P} \approx 10^{19} \mathrm{Gev}([1,[2,3,4])$. Typically in such theories the Planck energy is an invariant and particles can never exceed this energy. For example a photon cannot be blue-shifted over the Planck energy; likewise an hypothetical photon with energy $E_{P}$ would be seen with $E_{P}$ by all observers. These desirable properties ensure an invariant separation between the realms of classical and quantum gravity and provide an invariant cut off removing divergences from field theory.

But they also entail apparent paradoxes [5, 6]. Most infamously a naive application of the formalism leads to the conclusion that not only do single particles have a maximal energy $E_{P}$, but this property extends to collections of particles. Systems of particles, notably soccer balls, can blatantly exceed $E_{P}$; hence the eponymous problem.

There are a number of ad-hoc solutions to the "soccer ball problem" (see, eg. [3, 7] ). One approach posits that a system of particles simply does not have the same transformation laws and dispersion relations as its constituents [3], and that the distinction between elementary and composite is fundamental. Specifically it was proposed that a system of $N$ particles obeys non-linear transformations and non-quadratic dispersion relations obtained by replacing $E_{P}$ by $N E_{P}$ (or $l_{P}$ by $\left.l_{P} / N\right)$. This implies a non-associative addition law for energy and momentum, since one cannot "associate" terms in a sum thereby losing track of how many elementary particles it contains. The maximal energy for a system of $N$ particles is now $N E_{P}$, resolving the paradox.

Solving the soccer-ball problem ensures consistency with experiment, but also limits testability. In this paper we investigate whether there might be a middle ground where collections of particles retain the imprint of QG in a way that is not obviously inconsistent with experiment. For example, it could it be that loss of coherence is essential for solving the soccer ball problem. Perhaps a coherent superposition of particles (e.g. a laser) does have maximal energy $E_{P}$, and $\mathrm{QG}$ effects are of the order of the ratio between its total energy and $E_{P}$. The macroscopic objects we commune with in daily life are non-coherent, and perhaps only these are protected from soccer ball anomalies.

This speculation is not immediately at odds with experiment. Laser beams are the most powerful coherent superpositions of particles available, but the largest energy attained so far is about $10^{12} \mathrm{Gev}$, much smaller than $E_{P}[18,19]$. Laser technology is improving fast, however, so the possibility that a laser carrying a bulk energy close to $E_{P}$ might display strong $\mathrm{QG}$ behavior suggests a remarkable competitor to ultra high energy cosmic rays (UHECRs). These are widely regarded as the test bed of QG and DSR, because the predicted GZK energy cut off 8] probes Lorentz boosts at the highest available energies [9]. If the cutoff at $E \approx 10^{11} \mathrm{Gev}$ predicted by special relativity is present, however, then high energy cosmic rays will dry out as a probe of QG. Not so with Lasers. The currently operational NOVA laser [18] can deliver $400 \mathrm{KJ}$ of coherent light, i.e. $2.5 \times 10^{12} \mathrm{Gev}$, already larger than the highest cosmic rays detected. The planned National Ignition Facility [19] will raise this figure to $2.5 \times 10^{13} \mathrm{Gev}$.

Regrettably this possibility is by no means a generic feature of DSR or QG. The arguments leading to the soccer-ball problem are purely kinematic and refer to classical point particles. In this paper we reevaluate the situation from a field theory perspective. In field theory realizations of DSR there should be classical plane wave solutions with wavenumber $k_{\mu}=(\omega,-\mathbf{k})$, constrained by 
deformed dispersion relations (with a deformation dependent on $l_{P} \omega$, say). Their amplitude $A$ further tunes the bulk energy $\mathcal{E}$ of the wave. These waves form our "soccer balls". Upon quantization the quanta have energy and momentum proportional to $\omega$ and $\mathbf{k}$, and therefore feel dispersion relations deformed according to $l_{P} \omega$. It does not follow that the wave's bulk energy $\mathcal{E}$ and momentum $\mathcal{P}_{i}$, dependent on amplitude $A$, must feel deformations according to $l_{P} \mathcal{E}$ as opposed to $l_{P} \omega$. Indeed we show that it is very difficult to have a soccer ball problem in a field theory formulation of DSR.

The plan of the paper is as follows. In Section [II we carry out, in the undeformed theory, the model calculation later to be performed in field theories representing DSR. We use the stress-energy tensor to evaluate the mass-shell relations for the wave's bulk $\mathcal{E}$ and $\mathcal{P}_{i}$, and contrast them with the dispersion relations for $k_{\mu}$. Then, in Section ஹw explain how DSR may be represented by higher order derivative (HOD) field theories. Although we have Lasers in mind we keep our arguments general and consider massless and massive particles. For massless particles we consider the possibility of an energy dependent speed of light 10, 11], but this is by no means necessary. For simplicity we ignore spin and examine a real scalar field, but the constructions presented may be easily generalized to any spin including spin 1 . We set up a Lagrangian formulation for HOD theories and derive their stress energy tensor, essential for the assessment of the macroscopic properties of the waves.

Equipped with these tools, in Section IV we examine two concrete examples of DSR theories, where $k_{\mu}$ has deformed transformation laws and dispersion relations. We find that the deformations felt by bulk quantities $\mathcal{E}$ and $\mathcal{P}_{i}$ depend on $l_{P} \omega$, not $l_{P} \mathcal{E}$. Thus, as long as $\omega$ remains well below $E_{P}$ the deformations felt by the bulk wave are very small, regardless of the total $\mathcal{E}$, and therefore there is no soccer ball problem. The argument does not rely on the details of second quantization, however in Section $\nabla$ we show how it is possible to quantize in such a way that the dispersion relations felt by a system of $N$ particles can be obtained by the replacement $l_{P} \rightarrow l_{P} / N$, thus proving the suggestion in [3] from first principles.

This conclusion exempts DSR models from a paradox, but does not satisfy our motivations. We therefore spend the rest of the paper seeking theories that might have phenomena akin to the soccer ball problem, preferably only in the case of coherent objects. Non-quadratic Lagrangian theories are considered in Section DI] They have the property that both quanta and bulk dispersion relations are dependent on the energy density, i.e. on $\mathcal{E} /\left(V E_{P}^{4}\right)$. Therefore they also don't display a soccer ball problem. In Section [VII we argue that this conclusion is unavoidable without appealing to an unorthodox second quantization. This is developed in Section VIII where an exotic second quantization procedure is presented (to be contrasted with that in Section $(\nabla)$, for which the quanta's mass-shell conditions are deformed according to $l_{P} \mathcal{E}$, where $\mathcal{E}$ is the energy of the coherent packet to which the quanta belong. This is undoubtedly unusual, but cannot be ruled out a priori. Thus we satisfy our experimental motivation. An overview is provided in the concluding Section.

Throughout this paper we use a metric with signature +--- . We choose units where in the low energy limit, $\hbar=c=G=1$. Whenever we consider frequency dependent functions $\hbar(\omega)$ or $c(\omega)$ these refer to dimensionless ratios with the low energy values of $\hbar$ or $c$.

\section{A MODEL CALCULATION}

The basic idea behind this paper is that plane waves (or wave packets) form excellent test tubes with which to probe the behavior of sets of coherent particles in deformed special relativity. We can use their amplitude to tune the number $N$ of particles they contain. We can then compute the stress-energy of the wave seen as a bulk and check directly what the dispersion relations are for a set of $N$ coherent particles. Such coherent superpositions of quantum particles in effect form classical waves and provide good models for lasers.

We start by illustrating what we're hoping to do by considering the undeformed theory, taking as a prototype a free field theory with Lagrangian

$$
\mathcal{L}=\frac{1}{2}\left[\partial_{\mu} \phi \partial^{\mu} \phi-m^{2} \phi^{2}\right] .
$$

As explained in the introduction we shall ignore the complications of spin (polarization), but the argument can easily be generalized. Variation with respect to $\phi$ leads to the Klein-Gordon equation

$$
\left[\partial_{\mu} \partial^{\mu}+m^{2}\right] \phi=0
$$

which accepts plane wave solutions

$$
\phi=A e^{-i x^{\mu} k_{\mu}}
$$

or, more precisely

$$
\phi=A \cos \left(x^{\mu} k_{\mu}\right)
$$

(we can only use the complex notation for the real field $\phi$ as long as we are dealing with linear operations). The quanta, or particles, in this theory have momentum $k_{\mu}=\left(\omega, k_{i}\right)=\left(\omega,-k^{i}\right)$ and satisfy quadratic dispersion relations

$$
\omega^{2}-\mathbf{k}^{2}=m^{2}
$$

The proposed exercise is now to work out the dispersion relations for a collection of these quanta, by evaluating the energy $\mathcal{E}$ and momentum $\mathcal{P}_{i}$ of the wave (3).

Noether's theorem tells us that we need to collect the surface terms (or full divergences) eliminated in going from (1) to (2) in order to identify the conserved currents associated with symmetries of the action. Specifically, 
translations $x^{\mu} \rightarrow x^{\mu}+\delta x^{\mu}$ induce field variations $\delta \phi=$ $\partial_{\mu} \phi \delta x^{\mu}$. These in turn induce a variation in the action given only by the full divergence

$\delta S=\int d x^{4} \partial_{\mu}\left(\frac{\partial \mathcal{L}}{\partial\left(\partial_{\mu} \phi\right)} \delta \phi\right)=\int d x^{4} \partial_{\mu}\left(\frac{\partial \mathcal{L}}{\partial\left(\partial_{\mu} \phi\right)} \partial_{\nu} \phi\right) \delta x^{\nu}$

assuming that the field equations are satisfied. But the action does not depend explicitly on $x^{\mu}$ so another way to obtain this variation is to replace the fields by their explicit functions of $x^{\mu}$ in order to identify $\mathcal{L}(x)=$ $\mathcal{L}\left(\phi(x), \partial_{\mu} \phi(x)\right)$ and then compute

$$
\delta S=\int d x^{4} \frac{\partial \mathcal{L}}{\partial x^{\nu}} \delta x^{\nu}=\int d x^{4} \partial_{\mu}\left(g_{\nu}^{\mu} \mathcal{L}\right) \delta x^{\nu} .
$$

Comparing the two expressions we thus obtain the result that

$$
T_{\mu \nu}=\frac{\partial \mathcal{L}}{\partial\left(\partial^{\mu} \phi\right)} \partial_{\nu} \phi-g_{\mu \nu} \mathcal{L}
$$

is divergence free, that is $\partial_{\mu} T^{\mu \nu}=0$.

For theory (11) the stress-energy tensor is:

$$
T_{\mu \nu}=\partial_{\mu} \phi \partial_{\nu} \phi-g_{\mu \nu} \mathcal{L}
$$

Applying this expression to a wave we get

$$
\begin{aligned}
& T_{00}=\frac{A^{2}}{2}\left(\omega^{2}-\mathbf{k}^{2} \cos \left(2 x^{\mu} k_{\mu}\right)\right) \\
& T_{0 i}=\frac{A^{2}}{2} \omega k_{i}\left(1-\cos \left(2 x^{\mu} k_{\mu}\right)\right)
\end{aligned}
$$

(we should use the real expression (4) here rather than (3) since this operation is non-linear). Integrating over a sufficiently large volume (with respect to the wavelength) the oscillatory terms in these expressions vanish. Thus the wave's bulk energy $\mathcal{E}$ and momentum $\mathcal{P}_{i}$ inside a volume $V$ is given by

$$
\begin{aligned}
\mathcal{E} & =T_{00} V=\frac{1}{2} A^{2} \omega^{2} V \\
\mathcal{P}_{i} & =T_{0 i} V=\frac{1}{2} A^{2} \omega k_{i} V .
\end{aligned}
$$

Given that the wave's energy and momentum is the sum of its quanta's energy $\omega$ and momentum $k_{i}$, we learn that the number of quanta contained in the wave is

$$
N=\frac{1}{2} V A^{2} \omega
$$

so that

$$
\begin{aligned}
\mathcal{E} & =N \omega \\
\mathcal{P}_{i} & =N k_{i} .
\end{aligned}
$$

The basic assumption is that there is additivity when going from the (quantum) parts to the (classical, coherent) whole.
We can now check explicitly that the set of particles, i.e. the wave seen as a bulk, also satisfies quadratic dispersion relations,

$$
\mathcal{E}^{2}-\mathcal{P}^{2}=N^{2}\left(\omega^{2}-k^{2}\right)=(N m)^{2}
$$

The mass of the bulk equals the sum of masses of its quanta. Even though in this case we have considered a coherent superposition we see that the issue of coherence only affects the counting of $N$ in the wave, i.e. whether the amplitudes add or simply their squares.

None of this is surprising but it should be obvious that several steps in the calculation break down under deformed dispersion relations. We'll now try to repeat this "model calculation" in field theories representing deformed special relativity, in the hope of identifying dispersion relations for (coherent) collections of quanta. This should illuminate the soccer ball problem from a more fundamental perspective.

\section{HIGHER-ORDER FIELD THEORIES}

Before that, however, we need to develop some tools. A possible method for introducing deformed dispersion relations into field theory appeals to Lagrangians with higher order derivatives (see 12]). We'll need to generalize the methods for obtaining the Euler-Lagrange equation and the stress energy tensor for such theories. We stress that in what follows the variables $x^{\mu}$ are to be seen as standard (commutative) variables, and that this is to be contrasted with approaches based on noncommutative geometry $15,16,17$. The issue of invariance (and consequent energy dependence of the metric) is discussed in [12] and 14], and can be ignored at this level.

Consider an action where the Lagrangian depends on higher order derivatives

$$
S=\int d x^{4} \mathcal{L}\left(\phi, \partial_{\mu} \phi, \partial_{\mu \nu} \phi, \partial_{\mu \nu \alpha} \phi \cdots\right)
$$

Variation with respect to $\phi$ now leads to a much more complex structure of surface terms, since we have to convert terms of the form

$$
\frac{\partial \mathcal{L}}{\partial\left(\partial_{\mu \nu \ldots \beta} \phi\right)} \partial_{\mu \nu \ldots \beta} \delta \phi
$$

into terms proportional to $\delta \phi$. For a term involving $n$ derivatives of $\delta \phi$ this can be achieved by integrating by parts $n$ times, from which result $n$ full divergences. The final result entails the generalized Euler-Lagrange equations

$\frac{\partial \mathcal{L}}{\partial \phi}-\partial_{\mu} \frac{\partial \mathcal{L}}{\partial\left(\partial_{\mu} \phi\right)}+\partial_{\mu \nu} \frac{\partial \mathcal{L}}{\partial\left(\partial_{\mu \nu} \phi\right)}-\partial_{\mu \nu \alpha} \frac{\partial \mathcal{L}}{\partial\left(\partial_{\mu \nu \alpha} \phi\right)}+\cdots=0$

where we note that the sign alternates depending on the order of the derivative in each term. The divergences 
generated by this process have the form $\partial_{\mu} D^{\mu}$ with

$$
\begin{aligned}
D_{\mu}= & \frac{\partial \mathcal{L}}{\partial\left(\partial^{\mu} \phi\right)} \delta \phi+ \\
& \frac{\partial \mathcal{L}}{\partial\left(\partial_{\alpha}^{\mu} \phi\right)} \partial_{\alpha} \delta \phi-\partial_{\alpha} \frac{\partial \mathcal{L}}{\partial\left(\partial_{\alpha}^{\mu} \phi\right)} \delta \phi+ \\
& \frac{\partial \mathcal{L}}{\partial\left(\partial_{\alpha \beta}^{\mu} \phi\right)} \partial_{\alpha \beta} \delta \phi-\partial_{\alpha} \frac{\partial \mathcal{L}}{\partial\left(\partial_{\alpha \beta}^{\mu} \phi\right)} \partial_{\beta} \delta \phi+\partial_{\alpha \beta} \frac{\partial \mathcal{L}}{\partial\left(\partial_{\alpha \beta}^{\mu} \phi\right)} \delta \phi
\end{aligned}
$$

Following argument identical to the one in the previous section we can therefore identify the conserved stressenergy tensor

$$
\begin{aligned}
T_{\mu \nu} & =\frac{\partial \mathcal{L}}{\partial\left(\partial^{\mu} \phi\right)} \partial_{\nu} \phi+ \\
& \frac{\partial \mathcal{L}}{\partial\left(\partial_{\alpha}^{\mu} \phi\right)} \partial_{\alpha \nu} \phi-\partial_{\alpha} \frac{\partial \mathcal{L}}{\partial\left(\partial_{\alpha}^{\mu} \phi\right)} \partial_{\nu} \phi+ \\
& \frac{\partial \mathcal{L}}{\partial\left(\partial_{\alpha \beta}^{\mu} \phi\right)} \partial_{\alpha \beta \nu} \phi-\partial_{\alpha} \frac{\partial \mathcal{L}}{\partial\left(\partial_{\alpha \beta}^{\mu} \phi\right)} \partial_{\nu \beta} \phi+\partial_{\alpha \beta} \frac{\partial \mathcal{L}}{\partial\left(\partial_{\alpha \beta}^{\mu} \phi\right)} \partial_{\nu} \phi \\
& \cdots \\
& -g_{\mu \nu} \mathcal{L}
\end{aligned}
$$

It can be checked directly that the condition

$$
\partial_{\mu} T^{\mu \nu}=0
$$

is equivalent to the Euler-Lagrange equation (19). Since the position variables $x^{\mu}$ remain standard commutative variables, the concepts of translational symmetry and its associated conserved stress energy tensor remain unmodified. In particular the law (21) remains the same, except that the expression for $T_{\mu \nu}$ is much more complex. But the only novelties in this section are of a technical nature.

Higher order derivative (HOD) field theories may be used to represent modified dispersion relations and by extension DSR 12, 13]. The prescription is that the field equation should be obtained from the replacement

$$
k_{a} \rightarrow i \partial_{a}
$$

applied to whatever deformed dispersion relation,

$$
\omega^{2} f^{2}(\omega)-\mathbf{k}^{2} g^{2}(\omega)=m^{2}
$$

one wants to represent. Of course we may algebraically rearrange the dispersion relations before applying this prescription, thus leading to different field equations. This is the same ambiguity associated with the fact that the same dispersion relation may be represented by a variety of non-linear representations of the Lorentz group [3]. In practice the particular representation chosen fully fixes the field equation used.

For example, if the proposed particle kinematics is given by the invariant:

$$
\frac{\omega^{2}-\mathbf{k}^{2}}{1-\left(l_{P} \omega\right)^{2}}=m^{2}
$$

the field equation should be:

$$
\left[\frac{\partial_{\mu} \partial^{\mu}}{1+\left(l_{P} \partial_{0}\right)^{2}}+m^{2}\right] \phi=0
$$

This equation is higher than second order, is linear (i.e. accepts a superposition principle) and has plane wave solutions that satisfy dispersion relations (24). A less trivial matter is finding a Lagrangian from which (25) can be derived. One possibility is

$$
\mathcal{L}=-\frac{1}{2} \phi\left[\frac{\partial_{\mu} \partial^{\mu}}{1+\left(l_{P} \partial_{0}\right)^{2}}+m^{2}\right] \phi .
$$

More generally $\mathcal{L}$ can be obtained by sandwiching the deformed Klein-Gordon operator between two fields, but only if the dispersion relations contain no odd powers of $\omega$. This is equivalent to demanding that functions $f$ and $g$ in (23) are functions of $\omega^{2}$, or that their expansions in powers of $l_{P} \omega$ only has even powers. The dispersion relations are then symmetric under $\omega \rightarrow-\omega$, that is positive and negative frequencies (energies) are treated equally.

In terms of the field theory this requirement means that the Klein-Gordon operator should be real and only contain even order derivatives. If one begins with a dispersion relation which has odd powers of $l_{P} \omega$ one may still construct a Klein-Gordon operator and a Lagrangian as before; however the field equation derived from it will automatically be symmetrized, as will the dispersion relations it represents. We shall therefore assume that the dispersion relations have $\omega \rightarrow-\omega$ symmetry, even though this excludes some outstanding examples [2, 17].

As explained above, one may propose many different field theories corresponding to the same dispersion relations (but there is a one-to-one relation with the particular DSR or non-linear representation of the Lorentz group chosen). For example the expression (24) may be arranged as $\omega^{2}-\mathbf{k}^{2}=m^{2}\left(1-\left(l_{P} \omega\right)^{2}\right)$. Applying prescription (22) to these two equivalent expressions leads to inequivalent field theories; the latter is no more than a (linear, non-frequency dependent) redefinition of the units of frequency (or energy).

\section{THE ENERGY AND MOMENTUM OF WAVES}

Let us take as an example dispersion relations

$$
\frac{\omega^{2}-\mathbf{k}^{2}}{1-\left(l_{P} \omega\right)^{4}}=m^{2}
$$

which we assume belong to a non-linear representation of the Lorentz group with generators $K_{i}=U^{-1} L_{0 i} U$, where $L_{0 i}$ are the usual linear generators, and

$$
U\left(\omega, k_{i}\right)=\left(\omega\left(1+m^{2} l_{P}^{4} \omega^{2}\right)^{1 / 2}, k_{i}\right)
$$

(the procedure is described in detail in [3]). Then, we should rewrite the dispersion relations as

$$
\omega^{2}\left(1+m^{2} l_{P}^{4} \omega^{2}\right)-\mathbf{k}^{2}=m^{2},
$$


before applying prescription (22). This leads to the field equation

$$
\left(\partial_{\mu} \partial^{\mu}+m^{2}-l_{P}^{4} m^{2} \partial_{0}^{4}\right) \phi=0
$$

which indeed has plane wave solutions with a $k_{\mu}$ satisfying dispersion relations (27). A possible Lagrangian (giving this equation via (19)) is

$$
\mathcal{L}=\frac{1}{2}\left[\partial_{\mu} \phi \partial^{\mu} \phi-m^{2} \phi^{2}+l_{P}^{4} m^{2}\left(\partial_{0}^{2} \phi\right)^{2}\right]
$$

for which the stress energy tensor, computed according to (20), is

$$
T_{\mu \nu}=\partial_{\mu} \phi \partial_{\nu} \phi+l_{P}^{4} m^{2} \delta_{\mu}^{0}\left(\ddot{\phi} \partial_{0 \nu} \phi-\dddot{\phi} \partial_{\nu} \phi\right)-g_{\mu \nu} \mathcal{L} .
$$

When evaluated for a plane-wave, and integrated over a sufficiently large box (as done in Section III), this leads to bulk energy and momentum

$$
\begin{aligned}
\mathcal{E} & =T_{00} V=\frac{1}{2} A^{2} \omega^{2} V\left(1+2 l_{P}^{4} m^{2} \omega^{2}\right) \\
\mathcal{P}_{i} & =T_{0 i} V=\frac{1}{2} A^{2} \omega k_{i} V\left(1+2 l_{P}^{4} m^{2} \omega^{2}\right) .
\end{aligned}
$$

At once we see that the soccer ball problem has been eliminated. Consider a case where the quantum particles are definitely sub-Planckian, with $\omega \ll E_{P}$ and $m \ll E_{P}$. Then regardless of how large $\mathcal{E}$ is, we have

$$
\mathcal{E}^{2}-\mathcal{P}^{2} \approx(N m)^{2}
$$

with $\mathcal{E}=N \omega$ and $\mathcal{P}_{i}=N k_{i}$, and

$$
N \approx \frac{1}{2} V A^{2} \omega
$$

This is true even if $\mathcal{E} \gg E_{P}$, thus eliminating the soccer ball problem. Notice that $N$ here does not need to be the actual number of quanta, a matter to be refined in Sections $\nabla$ and VIII Interpreting $N$ as the macroscopic parameter defined as $N=\mathcal{E} / \omega$, the point is that departures from (36) are a function of $l_{P} \omega$ and therefore negligible for subPlanckian frequencies. Regardless of the second quantization details (and what the actual number of particles is) there is no soccer ball problem.

Bearing laser physics in mind (see Introduction) we consider another example, where the dispersion relation for a massless particle is given by:

$$
\frac{\omega^{2}}{1-\left(l_{P} \omega\right)^{2}}-\mathbf{k}^{2}=0
$$

Such a dispersion relation entails a frequency dependent speed of light (see, e.g. [3, 10]). A varying speed of light has been considered in a variety of circumstances 11,20 , 21, 22], some of which cosmological in nature. Before applying (22) we rewrite these relations as

$$
\omega^{2}\left(1+l_{P}^{2} \omega^{2} \mathbf{k}^{2}\right)-\mathbf{k}^{2}=0
$$

so that we now get field equation

$$
\left(\partial_{\mu} \partial^{\mu}-l_{P}^{2} \partial_{0}^{2} \partial_{i}^{2}\right) \phi=0 .
$$

A Lagrangian giving this equation is

$$
\mathcal{L}=\frac{1}{2}\left[\partial_{\mu} \phi \partial^{\mu} \phi+l_{P}^{2}\left(\partial_{0} \partial_{i} \phi\right)^{2}\right]
$$

and so the relevant components of the stress energy tensor are

$$
T_{0 \nu}=\dot{\phi} \partial_{\nu} \phi+l_{P}^{2}\left(\partial_{0 i} \phi \partial_{i \nu} \phi-\partial_{i}^{2} \dot{\phi} \partial_{\nu} \phi\right)-g_{0 \nu} \mathcal{L} .
$$

Evaluated for a plane-wave, and integrated over a large volume so as to eliminate the oscillatory terms, we get

$$
\begin{aligned}
\mathcal{E} & =T_{00} V=\frac{1}{2} A^{2} \omega^{2} V \frac{1+\left(l_{P} \omega\right)^{2}}{1-\left(l_{P} \omega\right)^{2}} \\
\mathcal{P}_{i} & =T_{0 i} V=\frac{1}{2} A^{2} \omega k_{i} V \frac{1+\left(l_{P} \omega\right)^{2}}{1-\left(l_{P} \omega\right)^{2}}
\end{aligned}
$$

and once more we see that there is no soccer ball problem. If $\omega \ll E_{P}$, then these expressions reduce to the undeformed ones regardless of the amplitude, and so anomalous behavior is triggered by Planckian $\omega$, not by $\mathcal{E} \sim E_{P}$.

What happens in these two cases is actually very general. Due to the linearity of the field equation and the quadratic nature of the stress energy tensor, $\mathcal{E}$ and $\mathcal{P}$ will always be quadratic in amplitude, and be deformed by a function of $l_{P} \omega$ rather than a function of $l_{P} \mathcal{E}$. This immediately eliminates the soccer ball problem. This is true for general Lagrangians of the form

$$
\mathcal{L}=-\frac{1}{2} \phi\left[\partial_{\mu} \partial^{\mu} \sum c_{n}\left(l_{P} \partial_{0}\right)^{2 n}+m^{2}\right] \phi,
$$

of the form

$$
\mathcal{L}=\frac{1}{2} \partial_{\mu} \phi \partial^{\mu} \phi+\sum c_{n}\left(\left(l_{P} \partial_{0}\right)^{n} \partial_{i} \phi\right)^{2},
$$

or similar generalizations for other Lagrangians considered in the examples above. It is believed that some realizations associated with non-commutative geometry 17] can be cast as HOD field theories, and therefore they will fall under this category.

The fact that these theories naturally bypass the soccer ball problem doesn't mean that interesting bulk behavior is not present if the quanta are Planckian, i.e. if $\omega \approx E_{p}$. Take expressions (42) and (43) (or Eq. 49). For quanta with $\omega \approx E_{P}$ we see that for the same amplitude $A$ a wave carries a much larger density of particles (indeed this is infinite for $\omega=E_{P}$ ). The implication is that the same laser beam intensity can be reached with a much lower amplitude, or conversely it takes much more energy to excite a wave with a given amplitude when its color approaches the Planck frequency. 


\section{SECOND QUANTIZATION}

The field $\phi$ in the previous section is a classical field and its amplitude a $c$-number, not a creation/annilation operator. No mention of the quantization procedure has been made or is necessary. All we learn is that a macroscopic object (here a classical wave) with $\omega \ll E_{P}$ does not run into Planckian behavior even if $\mathcal{E}$ is comparable or larger than $E_{P}$. By itself this is a solution of the soccer ball problem; however, if we want to know in detail how bulk quantities are deformed as a function of $\mathcal{E}$ and $N$, and in particular whether the prescription $l_{P} \rightarrow l_{P} / N$ is correct, we need to know how the number of quanta is defined. Here we present a simple quantization procedure leading to the prescription in [3] .

The main remark is that if we adopt a quantization procedure in which the wave's energy and momentum satisfies $\mathcal{E}=N \omega$ and $\mathcal{P}_{i}=N k_{i}$ (i.e. Eqns. (14) and (15) ) then this is equivalent to the solution $l_{P} \rightarrow l_{P} / N$ proposed in [3]. Take the first example in Section [V] If (14) and (15) are correct then (13) is deformed as

$$
N=\frac{1}{2} V A^{2} \omega\left(1+2 l_{P}^{4} m^{2} \omega^{2}\right)
$$

and so

$$
\mathcal{E}^{2}-\mathcal{P}^{2}=N^{2}\left(\omega^{2}-k^{2}\right)=(N m)^{2}\left(1-\left(l_{P} \omega\right)^{4}\right)
$$

which can be rearranged into

$$
\frac{\mathcal{E}^{2}-\mathcal{P}^{2}}{1-\left(\frac{l_{P} \mathcal{E}}{N}\right)^{4}}=(N m)^{2} .
$$

By comparing with (27) we see that the wave, seen as a collection of particles, satisfies dispersion relations which can be obtained from the quanta's relations by replacing the mass with the sum of the quanta's masses and $l_{P}$ by $l_{P} / N$, as intuited in [3]. The same is true of the second example; if Eqns. (14) and (15) are true then

$$
N=\frac{1}{2} V A^{2} \omega \frac{1+\left(l_{P} \omega\right)^{2}}{1-\left(l_{P} \omega\right)^{2}}
$$

and again we can derive

$$
\frac{\mathcal{E}^{2}}{1-\left(\frac{l_{P} \mathcal{E}}{N}\right)^{2}}-\mathcal{P}^{2}=0
$$

to be contrasted with (37).

This conclusion is quite general and therefore the veracity of the prescription $l_{P} \rightarrow l_{P} / N$ depends on whether we can implement a second quantization procedure for which Eqns. (14) and (15) hold true, with $N$ standing for the actual quantum number operator (which should have an integer spectrum). For the amplitudes to become proper creation and annihilation operators they must satisfy

$$
\left[a_{\mathbf{k}}, a_{\mathbf{k}^{\prime}}^{\dagger}\right]=\delta_{\mathbf{k k}^{\prime}}
$$

Then $N_{\mathbf{k}}=a_{\mathbf{k}}^{\dagger} a_{\mathbf{k}}$ is indeed the number operator: it has an integer spectrum and "counts" the number of quanta present in states belonging to a Fock space set up with $a^{\dagger}$ as usual. This is merely a definition, its physical content residing in the expression linking fields and amplitudes

$$
\phi=\sum_{\mathbf{k}} \frac{1}{\sqrt{2 V C(\omega)}}\left[a_{\mathbf{k}} e^{-i k \cdot x}+a_{\mathbf{k}}^{\dagger} e^{i k \cdot x}\right]
$$

or, equivalently, in the "convenience factor" $C(\omega)$ in this expression. In the undeformed theory $C=\omega$, a fact that follows from canonical quantization, but here we shall leave it as a free function and then investigate the implications of different choices.

Specifically we want to know if it is possible for the quantum Hamiltonian and mometum to be given by

$$
\begin{aligned}
H & =\sum_{\mathbf{k}} \omega N_{\mathbf{k}} \\
\hat{P}_{i} & =\sum_{\mathbf{k}} k_{i} N_{\mathbf{k}},
\end{aligned}
$$

essentially the required conditions (14) and (15). Even though there are ambiguities in defining conjugate momenta in HOD theories, we can certainly define the quantum Hamiltonian as

$$
H=\int d^{3} x T_{00}
$$

where $T_{00}$ is to be read off from (32) (and similarly for the momentum using $T_{0 i}$ ). The field $\phi$ in this expression is to be replaced by its quantum version (52), under a normal ordering prescription. The result is

$$
H=\sum_{\mathbf{k}} \frac{\omega^{2}}{C(\omega)}\left(1+2 m^{2} l_{P}^{4} \omega^{2}\right) N_{\mathbf{k}}
$$

and thus it is possible to realize (54) if we choose

$$
C(\omega)=\omega\left(1+2 m^{2} l_{P}^{4} \omega^{2}\right) .
$$

The question is now: to what canonical quantization procedure does this correspond? One view is that in HOD theories expressions (51) and (52) are more fundamental than the canonical quantization postulate

$$
[\phi(\mathbf{x}, t), \Pi(\mathbf{y}, t)]=i \delta(\mathbf{x}-\mathbf{y})
$$

where $\Pi$ is the momentum conjugate to $\phi$ (not simply defined for HOD theories). We point out that (51) and (52) with $C(\omega)$ given by (57) follow from canonical quantization (58) for a deformed momentum

$$
\Pi=\dot{\phi}-2 m^{2} l_{P}^{4} \dddot{\phi}
$$

Given the ambiguities in defining conjugate momenta for HOD theories, this proposal is certainly possible, if not 
unique. Notice that this calculation is only possible because there is no upper bound on $|\mathbf{k}|$, so that we still have the identity

$$
\sum_{\mathbf{k}} e^{i \mathbf{k} \cdot \mathbf{x}}=V \delta(\mathbf{x})
$$

In theories where there is a minimal wavelength as well as a maximum energy, this is no longer true and therefore (58) can never be realized. The view that (51) and (52) are more fundamental to second quantization is probably more sensible in such cases.

In this example we have worked backwards, but such a construction is always possible (as long as there is no upper bound on $|\mathbf{k}|)$. For the second example in Section IV the momentum conjugate to $\phi$ should be chosen as

$$
\Pi=\partial_{0}\left(1-2 l_{P}^{2} \partial_{i}^{2}\right) \phi
$$

or even

$$
\Pi=\partial_{0} \frac{1+\left(l_{P} \partial_{0}\right)^{2}}{1-\left(l_{P} \partial_{0}\right)^{2}} \phi
$$

to obtain similar results. In either case (58) leads to (51) and (52) with

$$
C(\omega)=\omega \frac{1-\left(l_{P} \omega\right)^{2}}{1+\left(l_{P} \omega\right)^{2}}
$$

and it can be checked that (54) is correct, ensuring (14) and (15). The bulk dispersion relations therefore satisfy the rule that $l_{P}$ should be replaced by $l_{P} / N$, where the particle number $N$ has now been defined rigorously.

It is of course possible to follow other second quantization procedures, and a rather exotic one will be examined in Section VIII As the previous section shows, none of this affects the fact that HOD theories cannot have a soccer ball problem. However, something different but akin to the soccer ball problem may be found if an exotic second quantization is chosen, quite different from the one proposed in this section.

\section{NON-LINEAR FIELD THEORIES}

HOD theories may be criticized on a number of grounds. One may question the stability of the Cauchy problem or even suspect that such theories contain ghosts upon suitable field redefinitions. None of these criticisms has been definitely proved or disproved. Yet there are ways to introduce deformed dispersion relations into field theory that don't invoke higher order derivatives. The conceptual gains are paid for by heavy technical complexity, because such theories must be necessarily non-linear. It turns out that these theories are even better protected against the soccer ball problem than those considered in the previous Sections.
The idea is that $k_{\mu}$ should roughly correspond to $\partial_{\mu} \phi$ when setting up the Lagrangian. For example one may propose a Lagrangian of the form

$$
\mathcal{L}=\frac{1}{2}\left[\frac{\partial_{\mu} \phi \partial^{\mu} \phi}{1+\left(l_{P}^{2} \partial_{0} \phi\right)^{2}}-m^{2} \phi^{2}\right]
$$

for theory (24), or of the form

$$
\mathcal{L}=\frac{1}{2}\left[\partial_{\mu} \phi \partial^{\mu} \phi-m^{2} \phi^{2}\left(1-\left(l_{P}^{2} \partial_{0} \phi\right)\right)^{4}\right]
$$

for theory (29). In general these theories have a Lagrangian that is not quadratic in the fields. NonQuadratic Lagrangians (NQL) introduce non-linearities into the field equation and therefore one loses the superposition principle.

For definiteness we consider as an example a theory with Lagrangian

$$
\mathcal{L}=\frac{1}{2}\left[\partial_{\mu} \phi \partial^{\mu} \phi-m^{2} \phi^{2}\left(1-\left(l_{P}^{2} \partial_{0} \phi\right)\right)^{2}\right]
$$

for which the field equation is

$$
\left[\partial_{\mu} \partial^{\mu}+m^{2}\right] \phi+m^{2} l_{P}^{4}\left(\phi \dot{\phi}^{2}+\phi^{2} \ddot{\phi}\right)=0 .
$$

Treating the last two terms (let's call them $\delta s$ ) as a perturbation, and trying out a solution of the form

$$
\phi=A \cos (\omega t-k x)+\delta \phi
$$

we find that to leading order the extra terms are

$$
\delta s=-\frac{1}{2} m^{2} l_{P}^{4} A^{3} \omega^{2}(\cos \Phi+\cos 3 \Phi)
$$

where the phase $\Phi$ is the usual $\omega t-k x$. From the terms in $\cos \Phi$ we thus find the condition

$$
\frac{\omega^{2}-\mathbf{k}^{2}}{1-\frac{1}{2}\left(l_{P}^{2} A \omega\right)^{2}}=m^{2}
$$

that is, we discover that the dispersion relations are deformed with a controlling parameter of the order $l_{P}^{2} A \omega$. Thus in these theories the wave's amplitude has a direct effect on the quanta dispersion relations. This is due to the non-linearity of the theory.

But there is another effect, due to the $\cos 3 \Phi$ term in $\delta s$. This should be cancelled by a perturbation $\delta \phi$, also proportional to $\cos 3 \Phi$, specifically:

$$
\delta \phi=-\frac{A}{16}\left(l_{P} A \omega\right)^{2} \cos ((3(\omega t-k x)) .
$$

This is an instance of the so-called phenomenon of ringing: in addition to the fundamental mode, a higher harmonic is necessarily excited. In this case the higher harmonic has three times the frequency of the fundamental mode, and its amplitude is suppressed by a factor of $\left(l_{P} A \omega\right)^{2}$.

Expression (70) is an interesting result. It appears that in this theory the quanta's dispersion relation knows 
about the bulk wave and not just about the frequency of the quanta. However this is not quite a version of the soccer ball problem, because the relevant bulk wave parameter is not its energy $\mathcal{E}$ but the energy density $T_{00}=\mathcal{E} / \mathrm{V}$. Indeed the rough condition for anomalous behavior is

$$
A^{2} \omega^{2} \sim T_{00} \sim \frac{\mathcal{E}}{V} \sim l_{P}^{-4}
$$

i.e. we need the energy density, rather than the total energy $\mathcal{E}$, to be Planckian. This is also the parameter controlling deformations of bulk behavior, e.g. $\mathcal{E}$ and $\mathcal{P}$. So it seems that both quantum and bulk behavior are controlled by the same parameter, which is in fact a feature of the bulk wave.

Condition (72) is even more restrictive than $\omega \sim E_{P}$, since we now need both the quanta's energy and their number density to be Planckian. In general we find that in NQL theories Planckian effects are more suppressed than in HOD theories, both in the quantum and bulk dispersion relations. However we have learnt an interesting lesson, as the next Section will highlight: it is possible for the quanta's properties to be controlled by a bulk feature.

\section{SOME ORIENTATION}

In this section we pause to summarize our findings so far. Having pointed out the experimental potential in the possibility that Planckian behavior is triggered by $\mathcal{E} \sim E_{P}$, we encountered severe difficulties in realizing this property in field theory. We investigated DSR implementations based on HOD Lagrangians:

$$
\mathcal{L}=\mathcal{L}\left(\phi, \partial_{\mu} \phi ;\left(l_{P} \partial_{0}\right)^{n} \phi\right)
$$

and on NQL

$$
\mathcal{L}=\mathcal{L}\left(\phi, \partial_{\mu} \phi ; \phi^{2}\left(l_{P}^{2} \partial_{0} \phi\right)^{n}\right) .
$$

We then distinguished between two levels of phenomenology, microscopic (relative to the quanta, and to $k_{\mu}$ ) and macroscopic (relative to the bulk wave, and $\mathcal{E}$ and $\mathcal{P}_{i}$ ). The first relates to dispersion relations for the quanta, i.e. the replacement

$$
\omega^{2}-\mathbf{k}^{2}=m^{2} \rightarrow q(\omega, k, m)=0
$$

where the deformation function $q$ depends on $l_{P}$ multiplied by some energy, not necessarily $\omega$. The latter relates to the behavior of matter in bulk, investigated here in the extreme case of a classical wave, made up of coherent quanta. In general we expect a deformation

$$
\mathcal{E}^{2}-\mathcal{P}_{i}^{2}=M^{2} \rightarrow Q(\mathcal{E}, \mathcal{P}, M)=0
$$

via a possibly different function $Q$ controlled by $l_{P}$ multiplied by some other energy, not necessarily $\omega$ or $\mathcal{E}$. We saw that in HOD theories

$$
\begin{aligned}
q & =q\left(\omega, k, m ; l_{P} \omega\right) \\
Q & =Q\left(\mathcal{E}, \mathcal{P}, M ; l_{P} \omega\right)
\end{aligned}
$$

whereas in NQL theories

$$
\begin{aligned}
q & =q\left(\omega, k, m ; l_{P}^{4} \frac{\mathcal{E}}{V}\right) \\
Q & =Q\left(\mathcal{E}, \mathcal{P}, M ; l_{P}^{4} \frac{\mathcal{E}}{V}\right) .
\end{aligned}
$$

Thus in HOD theories the Planckian nature of the quanta triggers both microscopic and macroscopic anomalies, whereas in NQL theories it is a bulk parameter, the energy density, that controls anomalous behavior (both microscopic and macroscopic). In either case the low energy world is well protected from the threat of Planckian effects.

But in the realm of abstract possibilities two interesting alternatives might occur. Firstly, anomalous macroscopic behavior might be triggered by a Planckian bulk energy $\left(\mathcal{E} \sim E_{P}\right)$, with

$$
Q=Q\left(\mathcal{E}, \mathcal{P}, M ; l_{P} \mathcal{E}\right)
$$

This is the soccer-ball problem. NQL theories, while not realizing this scenario, suggest an even more insidious second possibility. It could also be that

$$
q=q\left(\omega, k, m ; l_{P} \mathcal{E}\right)
$$

i.e., microscopic QG behavior might be triggered by a Planckian bulk energy (in the sense $\mathcal{E} \sim E_{P}$ ) where $\mathcal{E}$ is the energy of the coherent packet to which the quanta belong. As pointed out in the introduction, as long as either of these possibilities happens only for coherent collections of particles this is interesting rather than problematic.

The realization of either possibility in classical field theory is highly unlikely, however. It would require an integral-differential field equation, for example:

$$
\left[\partial^{\mu} \partial_{\mu}+m^{2}\right] \phi-l_{P} m^{2} \phi \int d^{3} x \dot{\phi}^{2}=0 \text {. }
$$

This equation approximately accepts plane-wave solutions, with dispersion relations

$$
\frac{\omega^{2}-\mathbf{k}^{2}}{1-l_{P} \mathcal{E}}=m^{2}
$$

But it would also entail severe non-locality: the value of the field at one point is directly entangled with the field everywhere else.

The turn of phrase just used points to an interesting new avenue. Perhaps the non-locality required to realize (79) or (80) is not present in the classical field theory, but is instead part of the quantization process, in a suitable alternative to Section D Quantum entanglement is non-local, and so is the phase coherence between all the quanta making up a laser. This may be just the "nonlocal" ingredient required by the anomalies (79) or (80). This we investigate in the next section. 


\section{ANOMALOUS SECOND QUANTIZATION}

In this Section we search for a quantization procedure that might lead to (79) or (80). Since we seek anomalous behavior with $\mathcal{E} \sim E_{P}$ but $\omega \ll E_{P}$, we may assume $\omega \ll$ $E_{P}$ and $\mathcal{E} / V \ll E_{P}^{4}$. Thus we may as well investigate anomalous quantization of an undeformed classical field theory (even if the considerations to follow in effect apply to HOD and NQL theories, and further anomalies will be present if $\left.\omega \sim E_{P}\right)$.

Since the undeformed Klein-Gordon equation is approximately valid, we have classical plane wave solutions $\phi=A \cos (\omega t-k x)$ with $k_{\mu}=\left\{\omega, k_{i}\right\}$ such that, approximately

$$
\omega^{2}-\mathbf{k}^{2}=m^{2} .
$$

The bulk quantities $\mathcal{E}$ and $\mathcal{P}_{i}$ satisfy:

$$
\begin{aligned}
\mathcal{E} & =\frac{V}{2} A^{2} \omega^{2} \\
\mathcal{P}_{i} & =\frac{V}{2} A^{2} \omega k_{i} \\
\mathcal{E}^{2}-\mathcal{P}^{2} & =(N m)^{2}=M^{2}
\end{aligned}
$$

with $N$ formally defined as before. All of this is purely classical.

In setting up second quantization in Section $\nabla$ we adopted a deformation of the usual factor $C(\omega)=\omega$ (c.f. Eqn. (52)), but the deformation was taken to be a function of the frequency. For all we know it could be a function of $l_{P} \mathcal{E}$ where $\mathcal{E}$ is the energy of the coherent packet under examination. Then,

$$
\phi=\sum_{\mathbf{k}} \sqrt{\frac{\hbar\left(l_{P} \mathcal{E}\right)}{2 V \omega}}\left[a_{\mathbf{k}} e^{-i k \cdot x}+a_{\mathbf{k}}^{\dagger} e^{i k \cdot x}\right]
$$

with

$$
\left[a_{\mathbf{k}}, a_{\mathbf{k}^{\prime}}^{\dagger}\right]=\delta_{\mathbf{k k}^{\prime}} .
$$

Once more $N_{\mathbf{k}}=a_{\mathbf{k}}^{\dagger} a_{\mathbf{k}}$ properly counts the number of quanta present in the states of the standard Fock space, set up with $a^{\dagger}$ as usual.

This construction is equivalent to an $\mathcal{E}$-dependent Planck constant (explaining the notation used). It follows from canonical quantization with

$$
[\phi(\mathbf{x}, t), \dot{\phi}(\mathbf{y}, t)]=i \hbar\left(l_{P} \mathcal{E}\right) \delta(\mathbf{x}-\mathbf{y}) .
$$

Mimicking the calculation performed in Section $\nabla$ we find that

$$
\begin{aligned}
H & =\sum_{\mathbf{k}} \hbar\left(l_{P} \mathcal{E}\right) \omega N_{\mathbf{k}} \\
\mathcal{P}_{i} & =\sum_{\mathbf{k}} \hbar\left(l_{P} \mathcal{E}\right) k_{i} N_{\mathbf{k}} .
\end{aligned}
$$

Thus additivity is preserved in the form:

$$
\begin{aligned}
\mathcal{E} & =N E \\
\mathcal{P}_{i} & =N p_{i}
\end{aligned}
$$

but now we find that the energy-momentum $p_{\mu}=$ $(E,-\mathbf{p})$ of the quanta is non-trivially related to their frequency and wavenumber:

$$
p_{\mu}=\hbar\left(l_{P} \mathcal{E}\right) k_{\mu} .
$$

We have so far ignored the distinction between the quanta's energy $E$ and their frequency $\omega$. We are now zooming in on this distinction, and implementing the anomalous element there.

But the dispersion relations for $k_{\mu}$ are undeformed (or rather, they're deformed according to $l_{P} \omega$ and we are taking the limit $l_{P} \omega \ll 1$ ). Thus the proposed deformation of the relation between $p_{\mu}$ and $k_{\mu}$ implies that the quanta's mass-shell condition must be deformed as a function of $l_{P} \mathcal{E}$. For example if

$$
\hbar=\frac{1}{1-l_{P} \mathcal{E}}
$$

so that

$$
\begin{aligned}
E & =\frac{\omega}{1-l_{P} \mathcal{E}} \\
p_{i} & =\frac{k_{i}}{1-l_{P} \mathcal{E}}
\end{aligned}
$$

then (83) leads us to the quantum mass-shell conditions

$$
\frac{E^{2}-p^{2}}{\left(1-l_{P} \mathcal{E}\right)^{2}}=m^{2}
$$

Therefore if we consider a high intensity laser, and perform experiments sensitive to the energy, rather than the frequency of its quanta (for example, photo-electric type experiments), we should become sensitive to QG. The most dramatic implication follows from the proved additivity (90), which implies

$$
N=\frac{1}{2} A^{2} \omega V\left(1-l_{P} \mathcal{E}\right)
$$

This creates a version of the soccer ball problem: we cannot build a coherent wave packet with an energy larger than $E_{P}$. As $\mathcal{E}$ approaches $E_{P}$ the packet behaves more and more like a single quantum, and the energy of a single quantum (for which now $E \approx \mathcal{E}$ ) cannot exceed $E_{P}$. In this limit we have blurred the distinction between bulk and quantum, thus creating a version of the soccer ball problem for coherent matter.

This construction has parallels with 13], but also striking differences. In that work it was proposed that

$$
p_{i}=k_{i} \hbar\left(l_{P} k\right) .
$$

leading to a deformed Heisenberg uncertainty principle and a frequency dependent Planck's constant. As in 13. we postulate that the relation between $p_{\mu}$ and $k_{\mu}$ is deformed, but as a function of $l_{P} \mathcal{E}$ rather than $l_{P} k$. Furthermore in [13] the quantum mass-shell relations are assumed to remain undeformed, implying deformed dispersion relations for $k_{\mu}$. We have exactly reversed this assumption. This is because HOD and NQL theories teach 
us that deformations of (83) can never be a function of $l_{P} \mathcal{E}$ (the purpose of this paper).

To restate the case in a more physical language, in 13 it takes more and more energy to produce a quantum as its wavelength approaches $l_{P}$. Here we suggested that it may take more and more energy to make a quantum (of whatever wavelength, not necessarily small), as the energy of the coherent packet to which it belongs approaches $\mathcal{E}_{P}$. Then $N \approx 1$ and the distinction between classical and quantum disappears. The packet behaves like a single quantum particle for which $E_{P}$ cannot be exceeded.

\section{CONCLUSIONS}

In DSR Planckian behavior is triggered whenever the energy of elementary particles approaches $E_{P}$. That Planckian behavior may also set in when the total energy in a collection is close to $E_{P}$ constitutes the "soccer ball problem". In this paper we pointed out that there is no obvious contradiction with experiment if only coherent superpositions of particles with collective energy close to $E_{P}$ display Planckian behavior. The proverbial "soccer balls" aren't coherent and the strongest lasers have bulk energies well below $E_{P}$.

Unfortunately, upon closer inspection, we found that in classical field theory realizations of DSR it is very difficult, if not impossible, not to solve the soccer ball problem. Our analysis was not exhaustive - for example we left out non-commutative field theory - but the results obtained are more than suggestive. We conclude that the soccer ball concern is a red herring, a mental block peculiar to simplistic kinematic models. With the community having spent so long worrying about the "problem", it is ironic that now when a "good side" is found, difficulties are encountered in realizing it at all.

But we found a better experimental window of oppor- tunity. Even though classical waves with sub-Planckian frequencies can never display anomalous bulk properties, it is possible for their quanta to behave unusually. We proposed that in sets of coherent particles the relation between the quanta's energy and frequency, and between their momentum and wavenumber, is deformed whenever the energy of the coherent packet nears $E_{P}$. In other words Planck's constant is deformed as a function of $l_{P} \mathcal{E}$ and, like most deformations considered in DSR, diverges for $\mathcal{E} \rightarrow E_{P}$. Then, in the same way that in DSR there cannot be elementary particles with energy bigger than $E_{P}$, it becomes impossible to construct a coherent packet with energy larger than $E_{P}$. As the packet energy gets larger each quantum absorbs more and more energy (for fixed frequency), until for $\mathcal{E} \rightarrow E_{P}$ the whole packet effectively becomes a single quantum, with sub-Planckian frequency, but Planckian energy. The distinction between bulk and particle then disappears, and it is impossible to push the energy of particle or packet beyond $E_{P}$. It is therefore impossible to have a Laser deliver more than about a TeraJoule of coherent light. The implied limitations to military systems, such as those inspired by SDI, are so tragic they shouldn't even be contemplated.

Once regarded as an embarrassment the soccer ball "problem" never exists in its classical field theory realization. But Pandora's box is opened with the realization that an important test, rather than a paradox, follows for a version of the phenomenon that only affects coherent collections of particles. We found that it is possible (but not generic) for the quantum mass-shell condition to be deformed as a function of $l_{P} \mathcal{E}$, where $\mathcal{E}$ is the total energy of a coherent wave packet. The consequent implications of high intensity Laser projects for QG phenomenology are highlighted for the first time in this paper.

Acknowledgements I'd like to thank Giovanni Amelino-Camelia, Kim Baskerville, Sabine Hossenfelder, Shahn Majid and Lee Smolin for very helpful comments on various versions of this manuscript.
[1] G. Amelino-Camelia, Nature 418:34-35,2002.

[2] J. Magueijo and L. Smolin, Phys. Rev. Lett. 88: 190403, 2002.

[3] J. Magueijo and L. Smolin, Phys. Rev. D67: 044017, 2003.

[4] J. Kowalski-Glikman, Phys. Lett. B547, 291-296, 2002.

[5] G. Amelino-Camelia, in "Karpacz 2001, New developments in fundamental interaction theories", 137-150; gr-qc/0106004

[6] G. Amelino-Camelia, Int.J.Mod.Phys. D11 (2002) 1643.

[7] S. Judes and M. Visser, Phys. Rev.D68: 045001, 2003.

[8] Takeda et al, Phys.Rev.Lett. 81 (1998) 1163

[9] G. Amelino-Camelia and T. Piran, Phys. Rev. D64: 036005, 2001.

[10] G. Amelino-Camelia et al, Nature 393 (1998) 763.

[11] J. Magueijo, Rept. Prog. Phys.66: 2025, 2003.

[12] D. Kimberly, J. Magueijo and J. Medeiros, Phys. Rev. D70, 084007, 2004.
[13] S. Hossenfelder et al, Phys. Lett. B 575, 85 (2003).

[14] J. Magueijo and L. Smolin, Class. Quant. Grav. 21: 17251736, 2004.

[15] J. Lukierski and A. Nowicki, Int. J. Mod. Phys.A18, 7-18, 2003, and references therein.

[16] G. Amelino-Camelia and S. Majid, Int.J. Mod. Phys. A15:4301-4324, 2000.

[17] J. Kowalski-Glikman, S. Nowak, Phys. Lett. B539: 126132, 2002.

[18] For an overview of Nova see "Energy Technology Review", Feb. 1985 (UCRL-52000-85-2, Lawrence Livermore National Laboratory, Livermore, California).

[19] "Energy Technology Review", Dec. 1994 (UCRL-52000094-12, Lawrence Livermore National Laboratory, Livermore, California).

[20] J. W. Moffat, Int. J. Mod. Phys. D 2, 351 (1993).

[21] A. Albrecht and J. Magueijo, Phys. Rev. D 59, 043516 (1999). 
[22] S. Alexander, R. Brandenberger and J. Magueijo, Phys.Rev.D67:081301,2003;
S. Alexander and J.
Magueijo, hep-th/0104093 